\title{
Conséquences de la chimiothérapie anticancéreuse sur la fertilité de la femme
}

\author{
Jean Paul GUASTALLA \\ Centre Léon Bérard, Lyon
}

\section{RESUME}

Le risque de stérilité par la chimiothérapie reste mal précisé, il est assez bien établi pour les alkylants, moins bien précisé pour les anthracyclines, le methotrexate et le fluoro-uracile et mal défini pour les alcaloïdes, les platines, l'etoposide, les taxanes. Les facteurs déterminants de la toxicité sont l'effet additif des drogues, les doses cumulées et l'âge des patientes. Le retentissement de la chimiothérapie sur la lignée germinale est étudié sur les cycles menstruels, les dosages hormonaux et les grossesses.

La chimiothérapie entraîne une destruction des ovocytes et des cellules de la granulosa. L'adriamycine entraîne chez la souris une apoptose ovocytaire qui pourrait être prévenue par une manipulation des signaux du cycle cellulaire (dérégulation du gène Bax, expression du gène antagoniste $\mathrm{Bcl-2}$, utilisation de sphingosine-1-phosphate ou de peptides inhibiteurs des caspases).

Les données cliniques de la littérature sont confuses et habituellement rétrospectives. A titre d'exemple la fertilité globale après chimiothérapie MOPP pour maladie de Hodgkin est de l'ordre de $20 \%$, les chimiothérapies adjuvantes du cancer du sein avec CMF, $F(A) C$ (fluorouracile anthracycline cyclophosphamide) ou TAC entraînent de 50 à $70 \%$ d'aménorrhées, la chimiothérapie de type PVB ou BEP pour tumeur germinale ovarienne altère relativement peu les possibilités de grossesses si un ovaire a pu être conservé et une majorité de femmes traitées par chimiothérapie pour maladie trophoblastique persistante avec methotrexate, actinomycine ou associations diverses restent fertiles.

La prévention de la fertilité est un enjeu thérapeutique majeur: les techniques de FIV sont rarement applicables en raison du retard qu'elles entraînent à la mise en route de la chimiothérapie; la conservation d'ovocytes est en phase de recherche ; les contraceptifs oestroprogestatifs n'ont pas montré d'efficacité, par contre les agonistes de GnRH pourraient être utiles, ils sont capables de prévenir la toxicité du cyclophosphamide sur l'ovogenèse chez la ratte et le singe macaque Rhésus et quelques études pilotes invitent à réaliser des études randomisées.
Mots clés : chimiothérapie, toxicité, fertilité, fonction ovarienne, agoniste $\mathrm{GnRH}$

\section{INTRODUCTION}

De plus en plus de femmes jeunes survivent après traitement de cancers grâce à une chimiothérapie (maladie de Hodgkin, Lymphomes, Leucémies, Ostéosarcomes, Tumeurs Germinales, Cancers du sein, Cancers de l'ovaire, Maladie Trophoblastique Persistante ...). Par ailleurs dans nos sociétés, le rythme de vie fait que souvent les femmes n'ont pas encore d'enfant à un âge relativement avancé et s'inquiètent au moment de la maladie du risque de ménopause lié à la chimiothérapie et de la possibilité de procréation ultérieure.

\section{GENERALITES}

Le risque de stérilité par la chimiothérapie est mal précisé, il est avéré pour les alkylants : busulfan, chlorambucil, cyclophosphamide, méchloréthamine, melphalan, procarbazine ; pour d'autres drogues le risque est possible mais moins bien établi : adriamycine, mitoxantrone, methotrexate, fluoro-uracile ; pour certaines le risque est mal défini : alcaloïdes, platines, etoposide, taxanes [4].

\section{Correspondance:}

Pr Jean-Paul GUASTALLA - Centre Léon Bérard, 28 rue Laënnec, 69008 Lyon - Tel 04.78.78.26.44 -

Email guastall@iyon.fnclcc.fr 
La toxicité de la chimiothérapie sur la fonction de reproduction dépend de la dose cumulée de médicament, ce qui représente le facteur déterminant, mais aussi d'un effet additif des drogues utilisées le plus souvent en association ; le rôle potentiel de la dose-intensité c'est-à-dire de la dose délivrée par semaine, n'est pas déterminé. II faut ajouter qu'en fonction du type de cancer, de l'âge des malades, leur état général et les tares associées, les protocoles de chimiothérapie sont nombreux et variés associant de multiples produits aux mécanismes d'action différents, administrés à des doses hétérogènes et adaptées en cours de traitement pour des durées variables et également adaptées ; les traitements varient également dans le temps au fil des progrès thérapeutiques ; les chimiothérapies dites intensives récemment utilisées sont particulièrement toxiques à tous les niveaux notamment sur le tissu de reproduction.

Ces raisons font qu'il est pratiquement impossible avant de débuter une chimiothérapie d'établir un pronostic fiable mais les malades doivent être informées du risque potentiel de stérilité.

\section{MOYENS D'ETUDE}

L'effet de la chimiothérapie sur la lignée germinale est évalué chez les malades par comparaison avec l'état de la fonction sexuelle avant la chimiothérapie ou bien avec la population générale de même âge, d'une part des cycles menstruels et des dosages hormonaux, d'autre part du nombre de grossesses. Les critères d'évaluation sont directs par la connaissance d'accouchements ou indirects par le nombre de grossesses, les cycles menstruels, le volume ovarien ou les dosages hormonaux.

\section{RESULTATS}

Les données de la littérature sont souvent confuses et habituellement rétrospectives. L'absence de définition homogène de l'aménorrhée et de l'état ménopausique rend compte des différences de résultats dans les publications [4]. Le suivi de cohortes est difficile du fait d'un long délai fréquent entre la chimiothérapie et le désir de grossesse.

De façon générale le tissu ovarien est plus résistant à l'effet néfaste de la chimiothérapie que le tissu testiculaire : chez 240 enfants de moins de 15 ans traités par chimiothérapie MOPP (Mechlorethamine Oncovin Procarbazine Prednisone) pour maladie de Hodgkin, une azoospermie est observée dans $83 \%$ des cas et une aménorrhée dans $13 \%$ [17], ce qui concorde avec les constatations faites chez l'adulte.

\section{Fertilité}

\section{a) Chimiothérapie pour maladie de Hodgkin et lympho- mes non hodgkiniens}

Globalement la fertilité moyenne après traitement de maladie de Hodgkin est de $21 \%(7 \%$ à $29 \%)$ et une conception est observée chez 30 à $60 \%$ des femmes non aménorrhéiques après traitement [8].

Le Tableau 1 montre, dans le traitement de la maladie de Hodgkin, l'importance de l'âge, du nombre de cycles de chimiothérapie et du type de chimiothérapie sur le taux d'aménorrhée : le risque est plus faible pour les femmes les plus jeunes, plus élevé quand le nombre de cycles est supérieur à 6 , et ce risque est nul pour 6 cycles d'ABVD (Adriamycine Bléomycine Vinblastine Dacarbazine), association considérée d'efficacité égale au classique MOPP ; ceci démontre le rôle du type de drogues utilisées.

Tableau 1 : Taux d'aménorrhée selon la chimiothérapie utilisée dans le traitement de la maladie de Hodgkin.

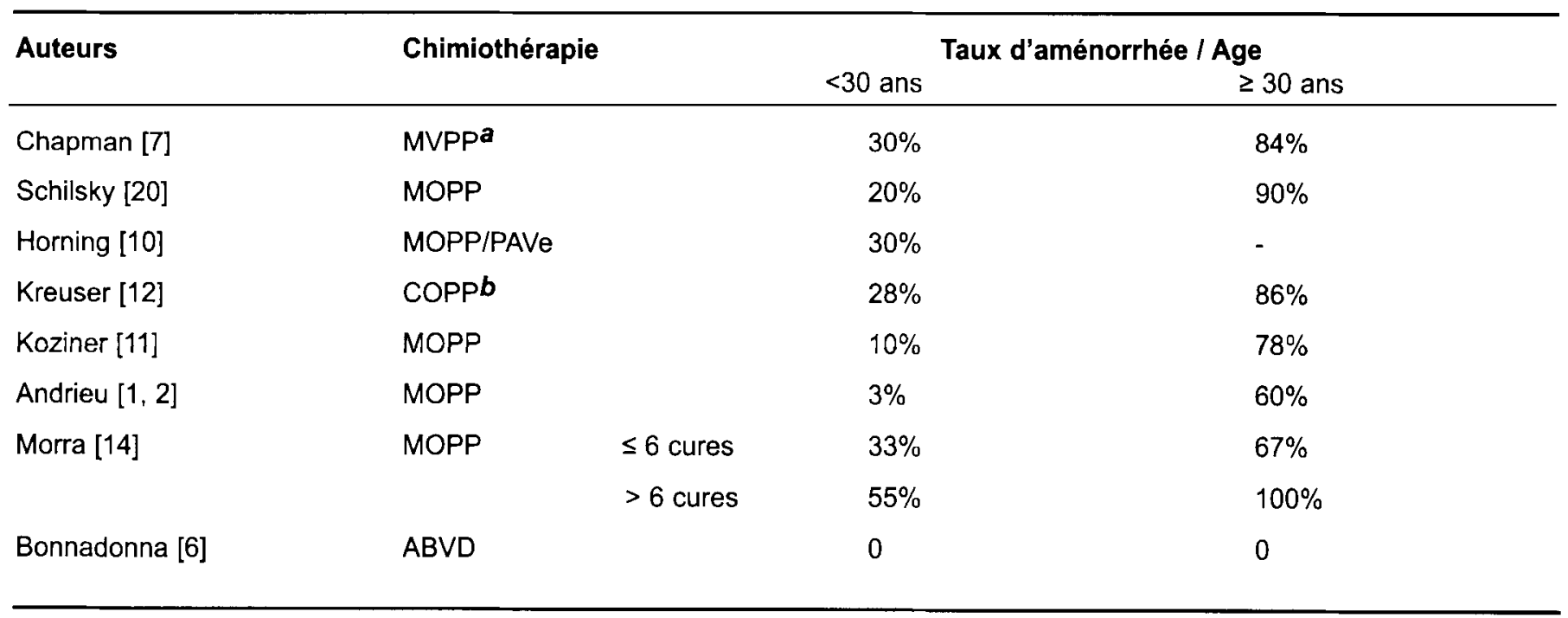

\footnotetext{
a : MVPP = Mechlorethamine Vinblastine Procarbazine Prednisone ;

b : COPP = Cyclophosphamide Oncovin Procarbazine Prednisone.
} 
Dans une autre série, sur 84 malades (âge médian 27 ans), traitées pour lymphome hodgkinien ou non hodgkinien, 34 malades (âge médian 31 ans), ont présenté une ménopause, 31 (âge médian 24 ans) ont pu concevoir, et 19 autres ont conservé une fertilité potentielle ; sur les 26 malades, âgées de 25 à 27 ans, ayant reçu une chimiothérapie intensive, 3 seulement ont pu concevoir [9] ; on constate là encore l'importance de l'âge et des fortes doses de chimiothérapie sur le risque d'infertilité.

\section{b) Cancer du sein}

Dans le cancer du sein les chimiothérapies adjuvantes entraînent une aménorrhée définitive dans 50 à $70 \%$ des cas selon le type de chimiothérapie et selon l'âge des malades : 3 à 24 cycles de la classique association CMF (Cyclophosphamide Methotrexate Fluorouracile) ont provoqué chez 3628 malades une aménorrhée dans $68 \%$ des cas : chez $40 \%$ des 713 malades de moins de 40 ans, et chez $76 \%$ des 1773 malades de plus de 40 ans [4]. Six cycles de l'association FAC (Fluorouracile Adriamycine Cyclophosphamide) d'utilisation plus récente ont entraîné une aménorrhée dans $54 \%$ des cas chez 359 malades et l'actuelle association TAC (Taxotere ${ }^{\circledR}$ Adriamycine Cyclophosphamide) administrée pour 6 cycles, dans $66 \%$ des cas chez 372 malades (Martin M., Pienkowski T., Mackey J., et al. Proceedings BC San Antonio, 2003).

\section{c) Cancer ovarien de la lignée germinale}

Chez 64 malades avec tumeur germinale ovarienne pour lesquelles la chirurgie a été conservatrice (utérus et un ovaire laissés en place) et traitées par chimiothérapie VAC (Vincristine Actinomycine Cyclophosphamide), PVB (Cisplatine Vinblastine Bléomycine) ou BEP (Bléomycine Etoposide Cisplatine), 38 enfants sont nés chez 29 femmes sur 38 ayant tenté une conception ; aucune anomalie n'a été observée chez les 16 enfants suivis dans cette série [21].

\section{d) Maladie trophoblastique persistante}

Environ $85 \%$ des femmes peuvent concevoir après chimiothérapie pour maladie trophoblastique persistante : il s'agit pour la plupart de femmes jeunes, de moins de 25 ans, traitées avec des drogues relativement peu toxiques sur le tissu ovarien : methotrexate ou actinomycine D utilisés seuls ou associés avec cyclophosphamide, etoposide, vincristine, cisplatine [8].

\section{Données biologiques}

Au niveau anatomique on observe une destruction par la chimiothérapie, des ovocytes et des cellules de la granulosa; cette destruction est moins massive que la destruction de la lignée spermatocytaire et des cellules de Leydig chez l'homme. Une destruction sélective des ovocytes pourrait être responsable d'une stérilité sans retentissement hormonal.

Sur le plan biologique, chez la souris, l'adriamycine déclenche une apoptose des ovocytes et un arrêt du cycle cellulaire des ovocytes fertilisés. Cette apoptose ovocytaire n'est pas reproduite chez les souris déficientes en facteur Bax, alors qu'elle est maintenue en cas de mutation de P53. L'apoptose adriamycine-dépendante est bloquée par la sphingosine-1-phosphate qui inhibe la voie céramide de promotion de la mort cellulaire, ainsi que par les peptides inhibiteurs des caspases (la caspase 2 notamment).

II est ainsi intéressant de noter que la destruction des ovocytes pourrait être prévenue par une manipulation des signaux de l'apoptose cellulaire : dérégulation du gène Bax qui apparaît comme un facteur clé, ou bien modulation du gène antagoniste $\mathrm{Bcl}-2$, ou encore utilisation de sphingosine-1-phosphate et de peptides inhibiteurs des caspases [19].

\section{PREVENTION DE LA STERILITE}

La prévention de la stérilité liée à la chimiothérapie est un enjeu thérapeutique majeur.

\section{Les contraceptifs oestroprogestatifs}

IIs n'ont pas montré d'efficacité en prévention.

\section{Les agonistes de GnRH}

Administrés simultanément à la chimiothérapie, les agonistes de $\mathrm{GnRH}$ pourraient limiter la toxicité ovarienne. Chez la souris, ils protègent la spermatogenèse de la toxicité du cyclophosphamide, et chez la rate et le singe macaque Rhésus ils protègent l'ovogenèse comme le montre l'expérience suivante : chez 6 singes femelles est réalisée, préalablement à la chimiothérapie, une ovariectomie unilatérale qui servira de témoin : 3 singes reçoivent simultanément au cyclophosphamide du leuprorelide ; après chimiothérapie ils conservent une FSH basse et la perte de follicules primordiaux est faible comparativement aux ovaires préalablement prélevés $(29 \% \pm 9 \%)$, alors que chez les 3 femelles n'ayant pas reçu d'agoniste de $\mathrm{GnRH}$, la FSH est augmentée et la perte de follicules primordiaux élevée $(65 \% \pm$ $3 \% ; p<0,05)[3]$.

En clinique trois études pilotes non randomisées suggèrent un effet protecteur potentiel des agonistes de $\mathrm{GnRH}$ :

a) La comparaison de 3 groupes de malades ayant reçu pour des cancers différents des chimiothérapies diverses (CAVPE, CVPP, ABVD, TAMO, ARA-C, MTT) montre que i) les 5 enfants pré pubertaires agés de 3 à 7 ans ont toutes par la suite des règles normales, ii) les 12 femmes âgées entre 15 et 20 ans ayant reçu un agoniste de GnRH au moment de la chimiothérapie gardent toutes des règles normales, et iii) deux grossesses ont été observées alors que les 4 femmes âgées entre 16 et 20 ans n'ayant pas reçu d'agoniste de $\mathrm{GnRH}$ sont toutes aménorrhéiques et présentent une hypoestrogénie [18].

b) Dans une autre étude, chez 24 femmes recevant une chimiothérapie adjuvante pour cancer du sein et du leuprolide : 12 ont reçu 4 AC (4 cycles d'adriamycine cyclophosphamide), $10: 4 \mathrm{AC}+4 \mathrm{~T}$ (4 cycles de paclitaxel $\left(\right.$ taxol $\left.\left.{ }^{\circledR}\right)\right), 1: 6$ CAF (6 cycles de cyclophosphamide adriamycine fluorouracile), et 1 : AT (adriamycine docetaxel (taxotere $\left.\left.{ }^{\circledR}\right)\right)+C M F$; une aménorrhée a été observé après 
3 cycles chez toutes les malades, 23/24 ont retrouvé des règles dans les 12 mois suivant la chimiothérapie, chez 15 les règles sont restées régulières, chez 5 irrégulières (dont 4 sous tamoxifène qui peut perturber les cycles menstruels) et six grossesses ont été observées chez 5 patientes (Fox K. R., Scialla J., Moore H. Proc. Am. Soc. Clin. Oncol., 2003 : abstr. 50).

c) Enfin chez 60 malades traitées par chimiothérapie et triptoreline pour lymphome ou maladie de Hodgkin on observe $5 \%$ de ménopause, comparativement à $55 \%$ de ménopause dans une série historique appariée de 60 malades n'ayant pas reçu d'agoniste de $\mathrm{GnRH}(p<0,05)$ (Tableau 2) [5]. Ces résultats doivent être interprétés avec prudence : les auteurs indiquent qu'il n'y a pas de différence entre les chimiothérapies administrées dans les deux groupes de malades sans rapporter le détail des traitements et recommandent finalement une étude randomisée pour confirmation des résultats.

Il a été montré par ailleurs que les analogues de $\mathrm{GnRH}$ inhibent la prolifération des cellules tumorales de cancer du sein, exprimant ou non des récepteurs hormonaux, ainsi que celle des cellules tumorales prostatiques [13], ce qui pourrait avoir un effet positif dans cette maladie ; les effets sur les cellules lymphomateuses ne sont pas précisés.

\section{La congélation d'embryons}

\section{a) La fécondation in vitro (FIV)}

Le délai des techniques de FIV est souvent non compatible avec celui de la mise en route de la chimiothérapie. La conservation d'ovocytes qui se heurte au même problème est encore en phase de recherche.

\section{b) Préservation de la fécondité par congélation d'em- bryons}

La congélation d'embryons pour conserver une fertilité après chimiothérapie se heurte d'une part comme on l'a signalé ci dessus, au délai d'obtention des ovules avant fécondation in vitro (de 15 à 45 jours), délai non compatible avec « l'urgence » de la chimiothérapie, et d'autre part à la nécessité d'un conjoint pas toujours présent au moment de la maladie.

Dans le cancer du sein, le déclenchement de l'ovulation par les médicaments usuels élève l'estradiolémie et risquent de stimuler la prolifération tumorale. Cependant une stimulation peut être réalisée élégamment par le tamoxifène, molécule de la classe des SERM (specific estradiol receptor modulator), qui inhibe la prolifération des cellules tumorales hormonosensibles et qui par ailleurs stimule l'ovogenèse. Douze patientes avec cancer du sein ont ainsi pris $40 \mathrm{mg} / \mathrm{j}$ de tamoxifène pendant 6 jours ; 12 embryons ont pu être congelés en 15 cycles, et 2 grossesses ont été obtenues à distance de la chimiothérapie chez 6 femmes ; la comparaison avec 5 cas de cancer du sein pour lesquels des embryons ont été congelés après ovulation naturelle démontre l'intérêt de la stimulation par tamoxifène qui permet d'obtenir plus d'ovocytes et plus d'embryons [15] (Tableau 3).

\section{Congélation d'ovocytes matures}

Cette technique reste expérimentale, sa mise au point permettrait de ne pas nécessiter la présence d'un conjoint au moment du traitement de la maladie cancéreuse.

\section{Congélation de tissu ovarien}

La congélation de tissu ovarien est encore expérimentale, mais pourrait être maîtrisée dans de brefs délais comme le suggère l'observation chez une femme de 30 ans traitée pour cancer du sein par chimiothérapie intensive à base de cyclophosphamide : la congélation d'un ovaire a été réalisée avant traitement, l'autre ovaire a été laissé en place ; comme attendu la chimiothérapie a entrainé une aménorrhée ; 6 ans plus tard des fragments de l'ovaire congelés sont greffés sous la peau de l'avant bras entrainant la restauration d'une fonction hormonale normale ; secondairement, après stimulation, une vingtaine d'ovocytes ont été recueillis à partir de l'ovaire greffé et l'injection intracytoplasmique de spermatozoïde a permis de réaliser l'implantation intra-utérine d'un embryon sélectionné au stade quatre cellules [16].

Tableau 2 : Fonction ovarienne et fécondité après chimiothérapie et administration d'un analogue de GnRH [5].

\begin{tabular}{lccc}
\hline & $\begin{array}{c}\text { Chimiothérapie } \\
\text { Triptoreline }\end{array}$ & $\begin{array}{c}\text { Chimiothérapie } \\
\text { (série témoin) }\end{array}$ & P \\
\hline Patientes évaluables/total & $58 / 60$ & $58 / 60$ & NS $^{\mathbf{a}}$ \\
Maladie de Hodgkin & 363 & 6 & NS \\
Lymphomes & 24 & 24 & NS \\
Age (ans) & $14-40$ & $14-40$ & NS \\
Radiothérapie & 36 & 35 & NS \\
Grossesses & $18(13$ femmes) & $13(8$ femmes $)$ & NS \\
Menstruations persistantes & 55 & 26 & $<0,01$ \\
Insuffisance ovarienne & $3(5 \%)$ & $32(55 \%)$ & $<0,01$ \\
\end{tabular}

a $:$ NS = non significatif. 
Tableau 3 : Fécondation In Vitro après stimulation de l'ovulation par tamoxifène [15].

\begin{tabular}{lccc}
\hline & Tamoxifène & $\begin{array}{c}\text { Fécondation In Vitro } \\
\text { Ovulation naturelle }\end{array}$ & P \\
\hline Age (ans) & 37,9 & 40 & NS $^{\mathbf{a}}$ \\
FSH de base $(\mathrm{mlU} / \mathrm{ml})^{b}$ & 11,5 & 9,5 & $\mathrm{NS}$ \\
Pic d'E2 $(\mathrm{pg} / \mathrm{ml})^{\boldsymbol{c}}$ & 442 & 278 & 0,006 \\
Nb total de follicules & 1,3 & 1,3 & $\mathrm{NS}$ \\
Follicules $>17 \mathrm{~mm}$ & 1,2 & 0,9 & 0,07 \\
Total ovocytes & 1,8 & 1,7 & $\mathrm{NS}$ \\
Ovocytes matures & 1,6 & 0,7 & 0,03 \\
Total embryons & 1,6 & 0,6 & 0,02 \\
\hline
\end{tabular}

$\boldsymbol{a}:$ : NS = non significatif $; \boldsymbol{b}^{\mathbf{b}}:$ Normale $<20 \mathrm{mlU} / \mathrm{ml} ; \boldsymbol{c}:$ Jour d' administration d'HCG.

\section{CONCLUSION}

La préservation de la fertilité est une préoccupation permanente pendant les chimiothérapies anticancéreuses. La toxicité ovarienne est imprévisible sur le plan individuel, cependant elle est plus fréquente avec certaines molécules (alkylants), plus fréquente avec les doses fortes de chimiothérapie et plus fréquente avec les traitements prolongés ; le risque augmente parallèlement à l'âge des malades. L'aménorrhée irréversible signe l'infertilité.

La prévention de la toxicité repose sur le choix des drogues les moins toxiques utilisées à des posologies et des durées optimales. L'administration à visée protectrice d'agonistes de GnRH doit être validée par des études randomisées, et l'utilisation de molécules modulant les signaux du cycle cellulaire est une voie de recherche. La fécondation in vitro est le plus souvent non envisageable en raison du retard au traitement qu'elle implique. La congélation d'ovules est encore au stade expérimental, ainsi que la conservation de tissu ovarien.

\section{REFERENCES}

1. ANDRIEU J.M., OCHOA-MOLINA M.E. : Pregnancies and offspring after MOPP therapy. Nouv. Presse Med., 1982, 11 : 3623-3625.

2. ANDRIEU J.M., OZANNE F., DANA M. et al. : Multiple chemotherapy (MOPP) followed by either focal or selective radiotherapy in clinical stages IA and II2A of Hodgkin's disease: results after four years of the use of prospective schedule $(H$ 7701) in 79 patients (author's trans). Bull. Cancer, 1981, $68: 217-223$.

3. ATAYA K., RAO L.V., LAWRENCE E., KIMMEL R. : Luteinizing hormone-releasing hormone agonist inhibits cyclophosphamide-induced ovarian follicular depletion in rhesus monkeys. Biol. Reprod., 1995, 52 : 365-372.

4. BINES J., OLESKE D.M., COBLEIGH M.A. : Ovarian function in premenopausal women treated with adjuvant chemotherapy for breast cancer. J. Clin. Oncol., 1996, 14 : 1718-1729.
5. BLUMENFELD Z., DANN E., AVIVI I., EPELBAUM R., ROWE J.M. : Fertility after treatment for Hodgkin's disease. Ann. Oncol., 2002, 13 (Suppl 1) : 138-147.

6. BONADONNA G., VALAGUSA P., SANTORO A., VIVIANI S., BONFANTE V., BANFI A. : Hodgkin's disease : the Milan Cancer Institute experience with MOPP and ABVD. Recent Results Cancer Res., 1989, $117:$ 169-174.

7. CHAPMAN R.M., SUTCLIFFE S.B., MALPAS J.S. : Cytotoxicinduced ovarian failure in women with Hodgkin's disease. I. Hormone function. J. Am. Med. Ass., 1979, 242 : 1877-1881.

8. CHAPMAN R.M. In : DeVita V.T., Hellman J.S., Rosenberg S.A. eds. Cancer : Principles and Practice of Oncology. 4th ed. Philadelphia, J.B. Lippincott, 1993.

9. FRANCHI-REZGUI P., ROUSSELOT P., ESPIE M. et al. : Fertility in young women after chemotherapy with alkylating agents for Hodgkin and non-Hodgkin lymphomas. Hematol. J., 2003, $4: 116-120$.

10. HORNING S.J., HOPPE R.T., KAPLAN H.S., ROSENBERG S.A. : Female reproductive potential after treatment for Hodgkin's disease. N. Engl. J. Med., 1981, 304 : 1377-1382.

11. KOZINER B., MYERS J., CIRRINCIONE C. et al. : Treatment of stages I and II Hodgkin's disease with three different therapeutic modalities. Am. J. Med., 1986, $80:$ 1067-1078.

12. KREUSER E.D., XIROS N., HETZEL W.D., HEIMPEL H. : Reproductive and endocrine gonadal capacity in patients treated with COPP chemotherapy for Hodgkin's disease. J. Cancer Res. Clin. Oncol., 1987, $113: 260-266$.

13. LOVAS S., PALYI I., VINCZE B., HORVATH J. et al. : Direct anticancer activity of gonadotropin-releasing hormone-lil. J. Pept. Res., 1998, 52 : 384-389.

14. MORRA E., LAZZARINO M., INVERARDI D. et al. : Therapyrelated ovarian dysfunction in women treated for Hodgkin's disease. Haematologica, 1986, $71:$ 209-215.

15. OKTAY K., BUYUK E., DAVIS O., YERMAKOVA I., VEECK L., ROSENWAKS Z. : Fertility preservation in breast cancer patients: IVF and embryo cryopreservation after ovarian stimulation with tamoxifen. Hum. Reprod., 2003, 18 : 90-95.

16. OKTAY K., BUYUK E., VEECK L. et al. : Embryo development after heterotopic transplantation of cryopreserved ovarian tissue. Lancet, 2004, $363: 837-840$. 
17. ORTIN T.T., SHOSTAK C.A., DONALDSON S.S. : Gonadal status and reproductive function following treatment for Hodgkin's disease in childhood : the Stanford experience. Int. J. Radiat. Oncol. Biol. Phys., 1990, $19: 873-880$.

18. PEREYRA P.B., MENDEZ RIBAS J.M., MILONE G. et al. : Use of $\mathrm{GnRH}$ analogs for functional protection of the ovary and preservation of fertility during cancer treatment in adolescents : a preliminary report. Gynecol. Oncol., 2001, 81 : 391-397.

19. PEREZ G.I., KNUDSON C.M., LEYKIN L., KORSMEYR S.J., TILLY J.L. : Apoptosis-associated signaling pathways are required for chemotherapy-mediated female germ cell destruction. Nat. Med., 1997, $3:$ 1228-1232.

20. SCHILSKY R.L., SHERINS R.J., HUBBARD S.M., WESLEY M.N., YOUNG R.C., DEVITA V.T. : Long-term follow up of ovarian function in women treated with MOPP chemotherapy for Hodgkin's disease. Am. J. Med., 1981, 71 : 552-556.

21. TANGIR J., ZELTERMAN D., MA W., SCHWARTZ P.E. : Reproductive function after conservative surgery and chemotherapy for malignant germ cell tumors of the ovary. Obstet. Gynecol., 2003, $101: 251-257$.

Communication au Colloque de la Fédération des CECOS, Lyon, 18 mars 2004.

Préserver la fertilité des patients soumis à des traitements anticancéreux : la cryopréservation des gamètes et du tissu gonadique.

Manuscrit reçu : mai 2004 ; accepté mai 2004.

\section{ABSTRACT}

Ovarian dysfunction in young cancer women who receive cytotoxic chemotherapy

Jean-Paul GUASTALLA

Sterility is a potential toxic effect of chemotherapy. This risk is well established for alkylating agents, but is less clearly defined for anthracyclines, methotrexate and fluorouracil and poorly defined for alkaloids, platinum, etoposide and taxanes. The main predictive factors for ovarian toxicity are the additive effect of cytotoxic drugs, the cumulative dose of each drug and the patient's age. This effect of chemotherapy is evaluated on menstrual cycles, hormonal assays and the number of pregnancies observed in patient cohorts.

Chemotherapy induces destruction of oocytes and granulosa cells. In mice, it has been shown that adriamycin may induce oocyte apoptosis, which can be prevented by modulation of cycle cell signalling (dysregulation of Bax gene or, on the contrary, expression of its antagonist gene Bcl-2 or inhibition of apoptosis with sphingosine-1phosphate or caspase inhibitors).
Clinical data in the literature are usually based on retrospective studies and are somewhat confused: global fertility after MOPP chemotherapy for Hodgkin's disease is about $20 \%$, adjuvant chemotherapy with CMF, F(A)C or TAC for breast cancer induces amenorrhea in $50 \%$ to $70 \%$ of cases, PVB or BEP chemotherapy for ovarian germ cell tumors has little effect on fertility when the uterus and one ovary can be preserved, and the majority of women treated with methotrexate, actinomycin $\mathrm{D}$ or various combinations for persistent trophoblastic disease remain fertile.

Preservation of fertility is a major goal for cancer patients receiving chemotherapy : in vitro fertilization could preserve the couple's fertility, but is usually not feasible as it would delay initiation of chemotherapy until after stimulation of ovulation; oocyte or ovarian tissue cryopreservation is at the stage of research; oral contraceptives have not been demonstrated to be effective to preserve ovarian function; gonadotropin releasing hormone (GnRH) agonists prevent cyclophosphamide toxicity in rat and monkey ovaries, and a few pilot clinical studies suggest that chemotherapy-induced amenorrhea could be prevented by administration of GnRH analogues simultaneously to chemotherapy, but randomised studies are necessary.

Key words : chemotherapy, toxicity, fertility, ovarian function, GnRH agonist 